
Pontifícia Universidade Cétólica $_{\text {do Rio de Janeiro }}$

Julia Teles da Silva

A pesquisa experimental para 0 desenvolvimento de técnicas em harmonia com o meio ambiente físico e social

Tese de doutorado

Tese apresentada como requisito parcial para a obtenção do grau de doutora em Design pelo Programa de pósgraduação em Artes \& Design da Pontifícia Universidade Católica do Rio de Janeiro

Orientadora: Jackeline Lima Farbiarz

Rio de Janeiro

Dezembro de 2014 


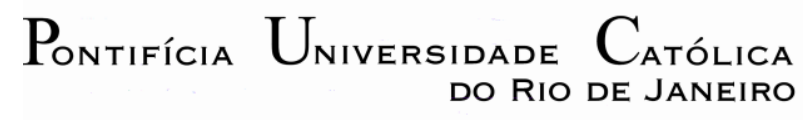

Julia Teles da Silva

\title{
A pesquisa experimental para o desenvolvimento de técnicas em harmonia com o meio ambiente físico e social
}

\begin{abstract}
Tese apresentada como requisito parcial para a obtenção do grau de doutora em Design pelo Programa de Pósgraduação em Design do Departamento de Artes e Design do Centro de Teologia e Ciências Humanas da PUC-Rio. Aprovado pela Comissão Examinadora abaixo assinada.
\end{abstract}

Professora Jackeline Lima Farbiarz

Orientadora

Departamento de Artes \& Design - PUC-Rio

Professora Nathalia Sá Cavalcante Departamento de Artes \& Design - PUC-Rio

Professor Ricardo Arthur Carvalho Departamento de Artes \& Design - PUC-Rio

Professor Luiz Eustáquio Moreira Departamento de Engenharia e Estruturas - UFMG

Professor Marcus Vinicius Dohmann Brandão Escola de Belas Artes - UFRJ

Prof ${ }^{a}$ Denise Berruzo Portinari. Coordenadora Setorial do Centro de Tecnologia e Ciências Humanas - PUC-Rio

Rio de Janeiro, 16 de dezembro de 2014 
Todos os direitos reservados. É proibida a reprodução parcial ou total do trabalho sem a autorização da universidade, da autora e da orientadora.

\section{Julia Teles da Silva}

Graduou-se em Comunicação Social na Universidade Federal Fluminense em 2007. Cursou três anos de Desenho Industrial na ESDI, UERJ. É mestre e doutora em Design pela PUC-Rio, tendo sido pesquisadora do LILD (Laboratório de Investigação em Living Design) e no LINC (Laboratório Linguagem, Interação e Construção de sentidos - Design)

Ficha Catalográfica

Silva, Julia Teles da

A pesquisa experimental para o desenvolvimento de técnicas em harmonia com o meio ambiente físico e social / Julia Teles da Silva ; orientadora: Jackeline Lima Farbiarz. - 2014.

184 f. : il. (color.) ; $30 \mathrm{~cm}$

Tese (doutorado)-Pontifícia Universidade Católica do Rio de Janeiro, Departamento de Artes e Design, 2014.

Inclui bibliografia 
Para meus pais, Branca Maria e Antonio Fernando, pelo incentivo aos estudos e ensinamentos. 


\section{Agradecimentos}

À minha orientadora, Jackeline Lima Farbiarz, por seu apoio, ensinamentos e incentivo. Ao professor José Luiz Mendes Ripper, que me orientou em parte da pesquisa.

À PUC-Rio, pelo auxílio concedido, estrutura e aulas. À Capes, pela bolsa de estágio de doutorado no exterior.

Ao professor David Rockwood, que orientou meu trabalho na Universidade do Havaí.

Aos colegas do LILD e do DeSSIn pela parceria e troca de conhecimentos.

Aos moradores da Aldeia Lago Lindo, pela receptividade e ensinamentos.

Aos organizadores do ShareFest e da Waihuena Farm, no Havaí, pela receptividade e colaboração na realização das oficinas.

Aos professores e funcionários do departamento de Artes \& Design da PUC-Rio, pelos ensinamentos e ajuda.

Aos funcionários do departamento de Arquitetura da Universidade do Havaí, pela ajuda e receptividade.

Aos professores Fernando José Fagundes Ribeiro e Ricardo Artur Pereira Carvalho, pelas contribuições na banca de qualificação.

À minha irmã Emilia Teles da Silva, pelo apoio e incentivo. Aos meus amigos Maria Rita Nepomuceno e Daniel Lôbo, pela parceria e pela ajuda na realização do vídeo.

Aos demais amigos, familiares e colegas, que, de alguma forma, me estimularam e ajudaram. 


\section{Resumo}

Silva, Julia Teles da; Farbiarz, Jackeline Lima. A pesquisa experimental para o desenvolvimento de técnicas em harmonia com o meio ambiente físico e social. Rio de Janeiro, 2015. 184p. Tese de doutorado. Departamento de Artes \& Design, Pontifícia Universidade Católica do Rio de Janeiro.

A tese investiga os princípios da pesquisa experimental para a criação de objetos em harmonia com o meio ambiente físico e social. A base para este estudo é a pesquisa realizada no Laboratório de Investigação em Livre Desenho (LILD) da PUC-Rio. Defendemos a tese de que a metodologia experimental para a pesquisa dessas técnicas se beneficia tanto de elementos da cultura material préindustrial, quanto de técnicas informatizadas e do conhecimento universitário moderno. A partir do encontro desses diferentes saberes, a pesquisa experimental pode enriquecer seus parâmetros e ter maior potencial para a geração de objetos em harmonia com o meio ambiente físico e social. A tese conta com o resultado da pesquisa em dois campos - uma aldeia indígena na Amazônia e o laboratório CPI (Construction Process Investigation), da Universidade do Havaí. Também é feita a descrição de oficinas realizadas para o compartilhamento de técnicas desenvolvidas no laboratório. A partir dos elementos, apresentamos um debate acerca da pesquisa e do desenvolvimento de objetos em harmonia com o meio ambiente físico e social e concluímos que o encontro entre os conhecimentos da cultura material de sociedades pré-industriais e da pesquisa acadêmica ligada à indústria potencializa a metodologia da pesquisa de técnicas em harmonia com o meio ambiente físico e social.

\section{Palavras-chave}

Pesquisa experimental; cultura; comunicação e interação; estruturas leves; materiais naturais; técnicas pré-industriais; sustentabilidade; pensamento sistêmico; convivencialidade. 


\section{Abstract}

Silva, Julia Teles da; Farbiarz, Jackeline Lima. The experimental research for the development of techniques in harmony with the physical and social environments. Rio de Janeiro, 2015. 184p. PhD Thesis. Art \& Design Department, Pontifícia Universidade Católica do Rio de Janeiro.

This thesis investigates the principles of the experimental research for the creation of objects that are in harmony with the physical and social environments. The base for this study is the research done in the Laboratório de Investigação em Livre Desenho (LILD). We defend the idea that the experimental methodology in this kind of research is enrichened both by elements of pre-industrial material culture and by modern academic knowledge. With these different knowledges, experimental research can enrich its parameters and have greater potential to create objects in harmony with the physical and social environments. Thus, the thesis has the result of two field researches - an indigenous village in the Amazon and the CPI (Construction Process Innovation) lab of the University of Hawaii. There is also a description of workshops that have been done to share the techniques developed in the laboratory. Based on these elements, we present a discussion about the experimental research and about the development of objects in harmony with the physical and social environments, and we come to the conclusion that the integration of elements of pre-industrial societies and of academic research related to the industry favors the development of the methodology for research of techniques in harmony with the physical and social environments.

\section{Keywords}

Experimental research; culture; communication and interaction; light structures; natural materials; pre-industrial techniques; sustainability; systems thinking; conviviality. 


\section{Sumário}

1. Introdução

2. Dos objetos para as relações

2.1 Introdução

23

2.2 Encontrando as bases de sustentação

25

2.3 Fundamentos apreendidos

26

2.3.1 Por uma abordagem sistêmica do Design 26

2.3.2 Pessoas, objetos e linguagem 32

2.3.3 Autonomia produtiva e convivencialidade 38

$\begin{array}{ll}2.4 \text { Considerações parciais } & 48\end{array}$

3. Princípios norteadores da pesquisa

experimental no LILD 50

$\begin{array}{ll}3.1 \text { Introdução } & 50\end{array}$

3.2. Princípio "modo de trabalho do laboratório" 56

3.2.1 O trabalho coletivo $\quad 57$

$\begin{array}{ll}3.2 .2 \text { O experimento prático } & 60\end{array}$

3.3 Princípio "Estruturas e materiais" 69

$\begin{array}{ll}\text { 3.3.1 Estruturas leves } & 69\end{array}$

$\begin{array}{ll}\text { 3.3.2 Os materiais pesquisados } & 74\end{array}$

$\begin{array}{ll}\text { 3.3.3 Sustentabilidade } & 87\end{array}$

3.3.4 $\mathrm{O}$ conhecimento de estruturas e materiais tradicionais 88 3.4 Princípio "difusão do conhecimento": 
O LILD e a educação - convivencialidade e autonomia produtiva 93

$\begin{array}{ll}3.5 \text { Considerações Parciais } & 100\end{array}$

4. Pesquisa de campo(s) 104

4.1. Introdução 104

$\begin{array}{ll}\text { 4.2. Metodologia } & 104\end{array}$

4.2.1. Pesquisa de campo(s), baseada em metodologia de observação participante 105

4.2.2. Análise de dados 106

4.3 Princípios norteadores da pesquisa experimental no CPI 107

$\begin{array}{ll}\text { 4.3.1 Introdução } & 107\end{array}$

4.3.2. Princípio "modo de trabalho do laboratório" 113

$\begin{array}{ll}\text { 4.3.2.1 O experimento prático } & 114\end{array}$

$\begin{array}{ll}\text { 4.3.2.2 O trabalho computadorizado } & 118\end{array}$

4.3.3 Princípio "Estruturas e materiais” 119

$\begin{array}{ll}\text { 4.3.3.1 Estruturas leves } & 119\end{array}$

$\begin{array}{ll}\text { 4.3.3.2 Os materiais pesquisados } & 121\end{array}$

$\begin{array}{ll}\text { 4.3.3.3 Sustentabilidade } & 122\end{array}$

4.3.3.4 O conhecimento de estruturas e materiais tradicionais 122

4.3.4 Princípio "difusão do conhecimento" 128

$\begin{array}{ll}\text { 4.3.5 Considerações parciais } & 129\end{array}$

4.4. Levantamento de dados Lago Lindo 131

$\begin{array}{ll}4.4 .1 \text { Introdução } & 131\end{array}$ 
4.4.2.1 O discurso huni kuin e a ausência de planejamento

4.4.2.2 O trabalho comunitário

4.4.2.3 Confecção de cerâmica

4.4.3 Estruturas e materiais

4.4.3.1 As casas

4.4.3.2 A riqueza material da floresta

4.4.4 Considerações parciais

4.5 Reflexões intermediárias sobre os campos

5. Experiências de ensino convivencial de estruturas amarradas de bambu

5.1 Oficina de treliça pantográfica de bambu em Waihuena Farm 151

5.2 Oficina de icosaedro de bambu no ShareFest, na Universidade do Havaí

6. Discussão dos resultados do levantamento de dados

7. Conclusão 